\title{
Towards developing a pocket therapist: an intelligent adaptive psychological support chatbot against mental health disorders in a pandemic situation
}

Intissar Salhi, Kamal El Guemmat, Mohammed Qbadou, Khalifa Mansouri

Laboratory of Signals, distributed systems, and artificial intelligence, ENSET, Hassan II University of Casablanca, Morocco

\begin{tabular}{l}
\hline Article Info \\
\hline Article history: \\
Received Apr 29, 2021 \\
Revised Jul 8, 2021 \\
Accepted Jul 14, 2021 \\
\hline
\end{tabular}

\section{Keywords:}

Chatbot

COVID-19

LSTM

NLP

Psychic disorders

RNN

Sequence-to-sequence

\begin{abstract}
Nowadays with COVID-19 ongoing epidemic outbreak, containment for weeks was one of the most effective measures adopted to deal with the spread of the virus until a vaccine could be efficient. Over that period, increased anxiety, depression, suicide attempts, and post-traumatic stress disorder are accumulated. Several studies referred to the need of using chatbots, which recognizes human emotions in such pandemic contexts. More recently, numerous research papers improved the ability of artificial intelligence methods to recognize human emotion. However, they are still limited. The aim of this paper is the development of a chatbot against the disturbing psychic consequences of the pandemic, taking human emotion recognition into account. The object is to help people; especially students; suffering from mental disorders, by progressively understanding the reasons behind them. This innovative chatbot was developed by using the natural language processing model of deep learning. An advanced model of deep learning has been elaborated the intention for people, and that to help them to regulate their mood and to reduce distortion of negative thoughts, that why a collection of a new database was done. The sequence-to-sequence model encoder and decoder consist of Long short-term memory cells, and it is defined with the bi-directional dynamic recurrent neural network packets.
\end{abstract}

This is an open access article under the $\underline{C C B Y-S A}$ license.

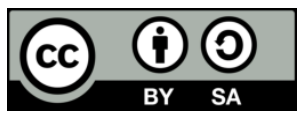

\section{Corresponding Author:}

Intissar Salhi

Laboratory of Signals, distributed systems, and artificial intelligence

ENSET, University Hassan II University of Casablanca

ENSETM, BP 159 Bd Hassan II Mohammedia 28000, Morocco

Email: salhi9477@gmail.com

\section{INTRODUCTION}

In a pandemic situation, the psychological reactions of the population are an essential factor of the virus spread [1]. Changes and countermeasures are taken to control it led to the appearance of emotional distress and/or mental health problems both during and after that pandemic [2]. Despite this fact, resources are not generally sufficient for managing and mitigating the impact that pandemics might otherwise cause to the mental health and wellbeing of the population [3]. While this may be understood during the acute stages of a pandemic when healthcare systems prioritize testing, transmission reduction, as well as critical patient care, the psychological and psychological requirements, cannot be neglected at any phase in pandemic management [4]. Mental Health stands for cognitive, emotional, and behavioral wellbeing [5], [6]. It is about how people are thinking, feeling, and behaving. It is the way people think, feel, and behave. As reported by the world health organization (WHO), the" mental health" term may be used sometimes as a term to indicate the absence of a mental disorder [7]. 
Recently, the important role of mental health in the achievement of global developmental challenges has been increasingly recognized, as illustrated by the inclusion of mental health in the goals of sustainable development [8]. One of the major causes of disabilities can be depression [9], [10]. Suicide remains the second cause of death among 15-to 29-year age-olds [11], [12]. People with serious mental health conditions are dying prematurely by up to two decades because of preventable physical impairment [13].

At the time of a pandemic, as with COVID-19, fear, anxiety, and stress may be considered as normal reactions towards the perceived and actual threats. When we are confronted with the uncertainty and struggles of the unfamiliar [14]. Aside from fear to contract this virus, there are also dramatic changes in our daily lives [15], due to the limited mobility required to sustain efforts aimed to contain and slowdown that virus's spread. Dealing with these new realities including home-schooling for children, telework, momentary unemployment, and the limited contact with friends, other family members, and colleagues; we must ensure that our mental and physical health, are well cared for.

One of the main technological solutions to the lack of staff in the field of mental health is chatbots [16]-[18], commonly known as conversational agents, conversational robots, or chatterbots [19]. Such systems can simulate human conversational skills via natural language [20]. At the acute stage of COVID-19, healthcare systems prioritized testing, transmission reduction, and critical patient care. Such conversational agents can be valuable tools, as they can substitute a therapist and can talk to people and provide some reassurance. Numerous trials have been carried out to design and implement conversational agents to support individuals in many areas of daily life, including healthcare, academia, commerce, finances, industry, and law [21]-[24]. They can process user inputs through natural language processing tools, then match these inputs with the intent and generate outputs [25]. They are programmed so that they can interact with users as a real person [26] and have the capacity to follow both context and word lists. Furthermore, we can classify them under three different classes, deep learning, end-to-end, and sequence-to-sequence chatbots [27]-[29].

The medical field is an area where chatbots are more and more adopted as a tool to ease information access by the patient and to reduce the workload on the clinician. psychotherapy has been the first discipline using conversational agents. It started in 1964 with ELIZA who was capable to simulate a psychotherapist by reformulating almost all the assertions of a patient into questions by asking him. This was done based on natural language processing [30]. However, the chatbot had some limitations, for instance, it was incapable of answering according to the context, and it was unable to handle the lost questions in any meaningful way. This is why he couldn't pass the Turing test [31]. By the year 1972, another chatbot known as Parry; was proposed by a researcher in the Stanford labs that were able of simulating an individual with Paranoiac Schizophrenia. Parry successfully passed the Turing test and had several conversations with ELIZA [32]. In recent years, as machine learning and artificial intelligence approaches are gradually being adopted in different application areas, advanced dialogue generation and management methods are increasingly using machine learning and deep learning techniques. A large number of commercial chatbot apps, offered as web or mobile-based solutions, were developed as a way to interact directly with patients. Some examples of healthcare chatbots include OneRemission1, which has been designed to assist cancer survivors, the Babylon Health, who is a symptom-checking tool, and Wysa [33], which is a mental health chatbot that interacts with the user to help him/her recognize signs of anxiety and depression [34], [35].

\section{THE PROPOSED METHOD}

The design of conversational agents requires personalization to suit the application's context. There are specific requirements when working on chatbot solutions. To deliver a technology that provides a service meeting user needs, one must consider the user demography, domain, interaction data and dialogue structure [36]. To improve the psychic condition of individuals in the COVID-19 scenario, the chatbot is here to improve the psychic state and thus must adapt itself to each person based on the previously mentioned features. Lately, artificial intelligence (AI) has made significant progress in medical fields, notably after the rise of neuronal networks and deep learning [37]. In this section, we are going the suggest a conversational agent architecture, define and explain its components, one by one in detail.

This research study introduces a therapist chatbot within COVID-19, that should support people suffering from mental disorders to eradicate negative thoughts affecting their mental health. This chatbot has been implemented with a sequence-by-sequence architecture with natural language processing. It is emotional and adaptive. Thus, capable of empathy and unexpected events. In this paper, we implemented a therapist chatbot as a tool to assist health care staff in the COVID-19 pandemic situation. This solution is based on recursive neural networks. The paper has been structured: The first section is a critical literature overview, while in the second section the materials and methods were presented that have used to generate 
the data set, to process and train it. The third section is dedicated to the implementation of the chatbot, results, and discussion, and finally to conclude and evoke some recommendations for future studies.

\subsection{The new approach of chatbot based on deep learning}

This paper offers a chatbot that provides a chat service for psychiatric monitoring. It collects and summarizes dialogues with textual data to recognize the user's feelings. Based on this data, the service's aim consists of developing personalized chat that emotionally conveys the user through text and voice. To achieve this goal, the methodology adopted is to first understand the context of the conversation, then the emotional recognition, and finally the generation of candidate responses adapted to the patient's situation. Section 3.2 describes the proposed architecture of the psychiatric chatbot.

\subsection{The proposed technical architecture}

This part provides the technical architecture of the chatbot based on deep learning. It consists of three major components that are: the natural language understanding unit, the dialogue management unit, and the natural language generating unit; which will be explained in details in the next section. Figure 1 shows the sequencing of those three units.

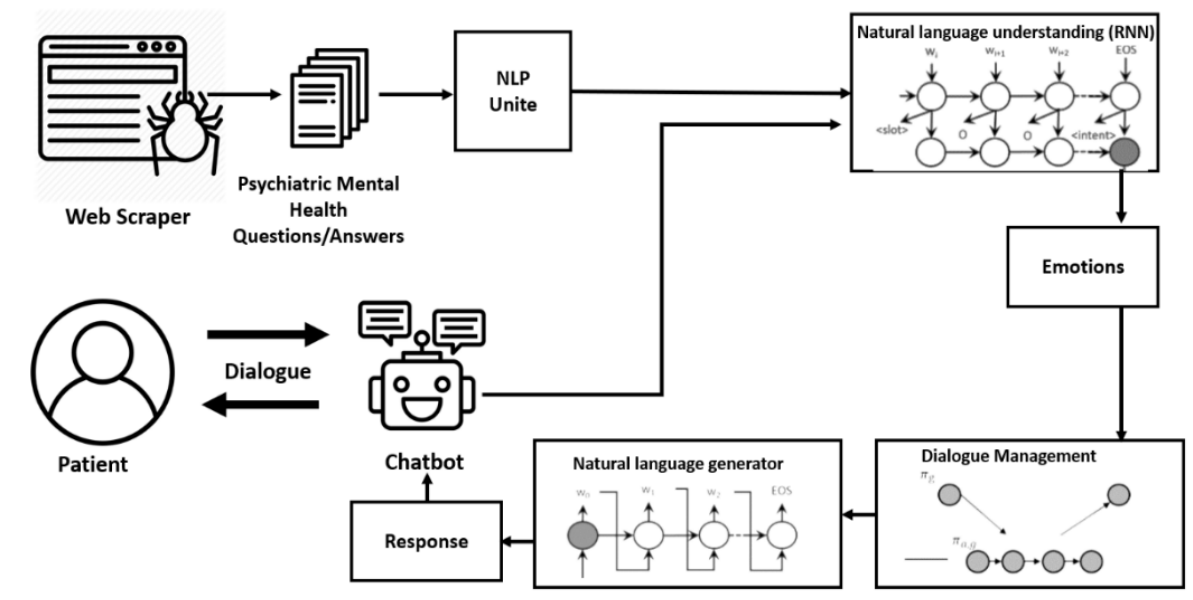

Figure 1. Deep learning-based psychiatric chatbot

Let's take an illustration to explain the way our chatbot works. Once the user puts a query or question to the chatbot, the chatbot takes charge of it, then the natural language processing unit (NLP) is activated. It picks up the request and splits it into understandable elements being the keywords on which it will focus. Afterward, he uses the natural language understanding unit (NLU) to compare the query to similar queries made in Reddit; which is a community website for social news in which people can ask questions and get answers; and tries to understand what the user is asking for. Once the chatbot has understood what the user wants to say, it scours the website to find the content that best answers the query. It extracts the relevant data and provides it in simple language using the natural language generation (NLG) unit. The user can then ask as many questions as they want and the process starts all over again. The advantage of machine learning is that, if the user regularly asks the same questions, the chatbot can start to anticipate his request and adapt to his case. The components of our architecture are described.

\section{RESEARCH METHODS}

In this section, all the units of the chatbot as well as the technologies and algorithms used in all of them will be describe.

\subsection{The natural language processing unit}

The first step for a user request is the pre-processing that takes place at the NLP unit. The preprocessing unit is charged by the NLP which consists of several stages, hence the named pipeline. The pipeline stages are mainly project-dependent. As our solution is based on Deep Learning, the text has to be converted into a spreadsheet. Therefore, the pipeline used in sentiment analysis is shown in Figure 2. And, units of the pipeline system as described hereafter. 


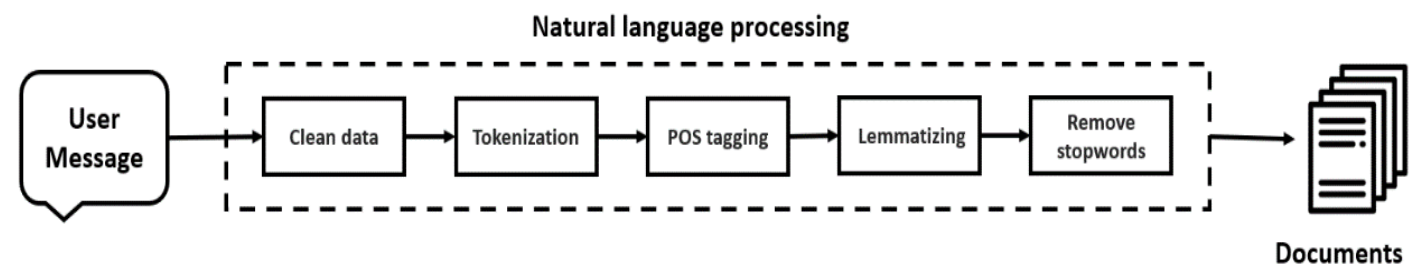

Figure 2. Natural language processing pipeline

a) Data Cleaning: Stemming refers to the generation of morphological variations of a root or base word. The filtering programs are commonly called filtering algorithms or "steamers". Stemming algorithms reduce words to stem terms. The stem is an important part of the process of pipetting in natural language processing. Stem input is done using symbolic words. Also, in this part punctuations are deleted because they are a useless addition and they do not add value or meaning to the NLP model.

b) Tokenization: In this cell, the converting process of a string of text into a list of tokens or elements is done. These tokens may be words that are elements of a sentence, as well as a sentence that is an element of a paragraph.

c) Post Tagging: Talking about marking the part of the speech, can be defined as the process of attributing a part of speech to a particular word. This is usually called part of speech marking (POS). In simple terms, POS tagging is a process of labeling all the words in a sentence with their corresponding part of speech. We already know that parts of speech include nouns, verbs, adjectives, pronouns, adverbs, conjunctions, and their subcategories.

d) Lemmatizing: Lemmatization refers to the process of combining the different inflected aspects of a word to be analyzed as one single element. Lemmatization works similarly to inflection, but it gives context to words. It thus connects words that have a similar meaning to the word.

e) Remove stop-word: A stop word is a commonly used word (such as "the", "a", "an", "in") that a search engine has been programmed to ignore, both when indexing entries for search and when retrieving them as a result of a search query. Indeed, these words should not occupy space in the database and should not waste processing time either. It is possible to eliminate them easily by storing a list of words considered as empty words.

\subsection{The natural language comprehension unit - detecting intent}

This unit is in charge of the conversion of the user's statement into a preselected semantic pattern under the system's requirements, i.e., in a system Understandable format. That includes a location-filling task with intention detection. For instance, the intention can be a greeting, it can be informative where the user gives extra text data [38]. The task of padding locations and intention detection is perceived as a sequence labeling problem. As a result, NLU is generally implemented as an LSTM-based recurrent neural network with a conditional random field (CRF) layer upward of it. The presented prototype is a Seq2Seq model utilizing a bi-directional LSTM neural network, that fulfills the intervals and predicts intention simultaneously. Alternatively, this model does the same thing by using the attention-based RNN. To complete this request, labels in the data set consist of the following: concatenated start - inside - outside (B - I - O) marker tags, intention marker tag, and auxiliary end of string (E-O-S) marker tag.

a) Long-term and short-term memory model

LSTM is a particular type of RNN that is suitable for temporal data or what we call sequential data. LSTMs preserve the information over time which implies that they can remember their previous inputs, this is why they are highly recommended for time-series predictions. with specific forgetting ports, besides the entry and exit ports of a simple RNN. LSTM is made to keep remembering the entry state for a longer duration than a single RNN, thus ensuring accuracy in the processing of long sequences. Figure 3 shows the layout of an LSTM cell.

b) The conditional random fields model (CRF)

The model CRF model $\mathrm{p}(\mathrm{y} / \mathrm{x}, \omega)$, used to predict the number of labels $(\mathrm{y})$ from the sequences of measurements (x), is given in (1), where the model parameters are given, $\psi(\mathrm{x}, \mathrm{y})$ is the chosen characteristic vector and $\mathrm{Z}(\omega, \mathrm{x})$ is the cumulative sum of $\mathrm{p}(\mathrm{y} / \mathrm{x}, \omega)$ for all possible y's. The parameters of this model $\mathrm{x}$ and $\mathrm{y}$ are learned from a training set. $(y, x)=\left\{x_{i}, y_{i}\right\}, i=1, \ldots, N$. With $\omega=\arg \max _{w}(y / x, \omega)$ the conditional maximum probability. Figure 4 is a representation of the LSTM-CR bidirectional attention model. 


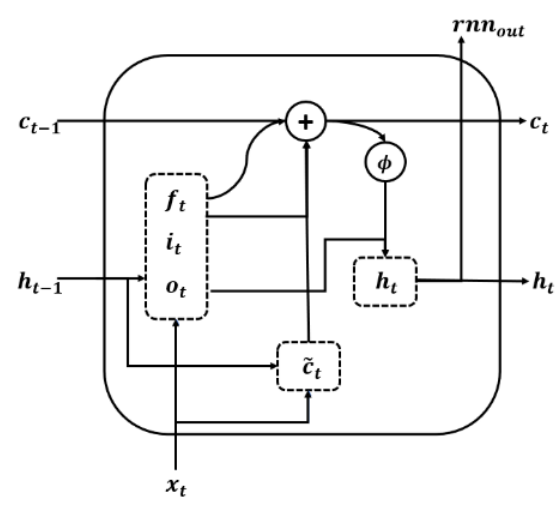

Figure 3. LSTM cell architecture

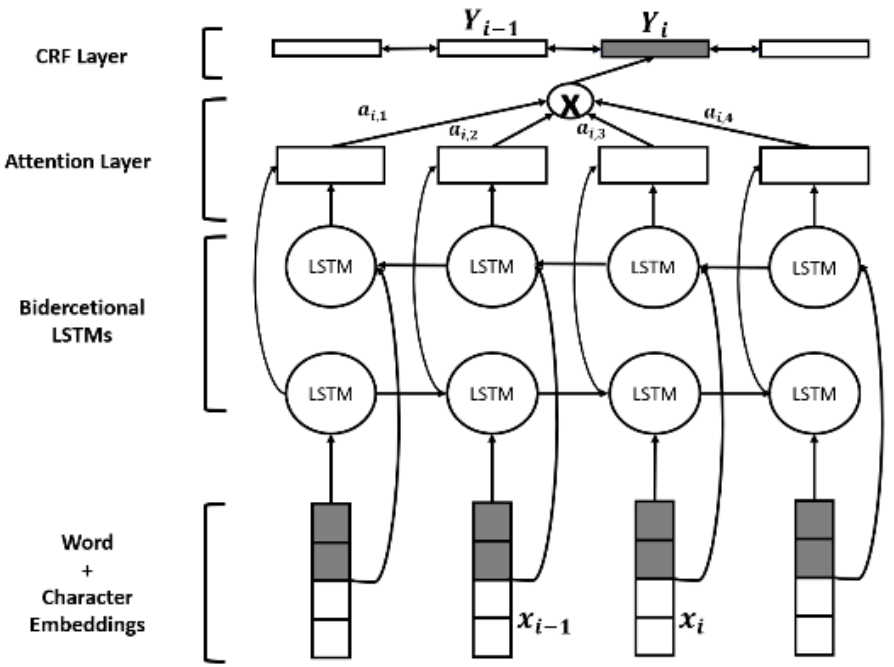

Figure 4. Representation of bidirectional LSTM-CRF attention model

$$
p(y / x, \omega)=\frac{\exp \left(\omega^{t} \psi(x, y)\right)}{Z(\omega, x)}
$$

\subsection{The natural language generation unit (NLG)}

This is where the generation of text from a representation of meaning happens. That process can be considered as the opposite of understanding the natural language. Within the NLG, it answers as a semantic framework, corresponding to a sentence in natural language, which is understood by the end-user. NLG components can be model-based, rule-based, or Hybrid In some scenarios. The rule-based NLG produces predefined model statements for a given semantic framework, so they are very limited and have no power of generalization. Even though several general-purpose rule-based systems have already been developed, these are often difficult to adapt to small task-oriented applications giving their generalization.

Machine-learning-based NLG systems have become increasingly used in today's dialog systems. These NLG systems use multiple sources as inputs, including the content map, which is a meaningful representation of what needs to be delivered to the user. A knowledge base, as a structured database that provides domain-specific characteristics. The User Model provides constraints to the output statement. The dialog history gives information on the previous rounds to prevent reference expressions and repetition.

\subsection{Dialog management (DM)}

The DM can be connected to an external database or a knowledge base to trigger more meaningful responses [39]. The dialogue manager has two components: Dialogue state tracking (DST) and policy learning that is the reinforcement learning agent (RL). DST is a complex and crucial component to correctly infer the state of conviction of dialogue, considering the whole history up to this point. Policy Learning is 
responsible for selecting the best action, i.e., the system's answer to the user's statement, to bring the user to reach its objective in a minimum number of dialogue cycles.

\subsection{Response generation: reinforcement learning with Seq2Seq}

Reinforced Learning is a field of machine learning. It involves taking appropriate actions to maximize rewards in a particular situation. Reinforcement learning is used by a variety of software and machines to find the best behavior or path in a specific situation. It differs from supervised learning in the sense that in supervised learning, the training data is accompanied by an answer key, so that the model is trained with the correct answer itself, whereas in reinforcement learning, there is no answer but the reinforcer decides what to do to accomplish the given tasks. In absence of a set of training data, however, it is expected to learn from its experience.

a) Recurrent neural network

A recurrent neural network (RNN) [40] is designed to preserve the previous state of neurons. This allows the neural network to preserve the context and produce a result based on the previous state. This approach makes RNNs desirable for chatbots, as it is essential to preserve the context of a conversation to understand the user.

b) Sequence to sequence model

The seq2Seq models [41] are based on RNN architecture and consist of two RNNs: one encoder and one decoder. While the encoder processes the input, the decoder deals with the output. These models can be considered as a decoder node that produces a corresponding output to an encoder node. This model is easy to use in machine translation because a word corresponding to the output language can be generated by the decoder easily by looking at only one word of the input language at a time. The following figure shows a simple sequence model. With $x_{i}$ is input sequence at step $\mathrm{I}, h_{i}$ the hidden state for each time step, and $y_{i}$ is output sequence at step i. Figure 5 is a representation of the sequence model.

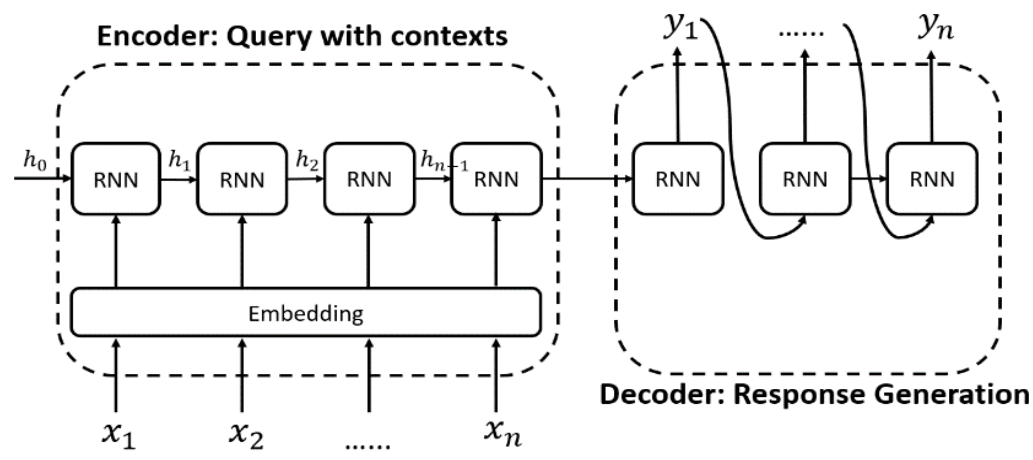

Figure 5. The sequence-to-sequence model structure

The encoder and the decoder are LSTM models (or sometimes GRU models). The encoder interprets the input sequence and synthesizes the data into what is known as internal state vectors as well as context vectors (in the case of the LSTM they are called the hidden state vectors or cell states). The encoder outputs are eliminated so that only the internal states are retained. The purpose of the context vector is the integration of data from all inputs to assist the decoder to make accurate predictions. The hidden states $h_{i}$ are calculated using the (2).

$$
h_{n}=f\left(w^{(h h)} h_{n-1}+w^{(h x)} h_{n x}\right)
$$

The LSTM reads the data, sequence after sequence. Thus, if the entry has a sequence of length "n", it is said that the LSTM reads it in time steps "n". The decoder is an LSTM with initial states initialized to the final states of the LSTM encoder, i.e., the context vector of the final encoder cell is entered into the first cell of the decoder network. By using these initial states, the decoder starts to generate the output sequence, and these results are also taken into account for future outputs. A pile of several LSTM units where predicts an 
output $y_{n}$ at time step n. Every recurring unit accepts a hidden state from the previous unit and generates and outputs its hidden state. Any hidden state $h_{i}$ is calculated using the (3).

$$
h_{n}=f\left(w^{(h h)} h_{n-1}\right)
$$

And the output $y_{n}$ at the time step $\mathrm{n}$ is calculated using the (4).

$$
y_{n}=\operatorname{softmax}\left(w^{S} h_{n}\right)
$$

The outputs are calculated by the use of the hidden state at the current time step and the respective weight $\mathrm{W}(\mathrm{S})$. Softmax is used to create a probability vector that will help to determine the final output (for example, it is a word in a question-answer problem) [42].

\section{RESULTS AND DISCUSSION}

The first step is the collection of data from the Reddit website, the aim of this step is to provide the Database, A fraction of the scrapping program code is displayed in Figure 6. This part is essential since a database that meets the requirements of this problem is not available. A selection of sub-directories (subReddit) has been made, to have only the questions and answers on mental disorders. The selected sub-Reddit are anxiety, depression, stress, depression_help, talk-therapy, askatherapist, as shown in the Figure 6,

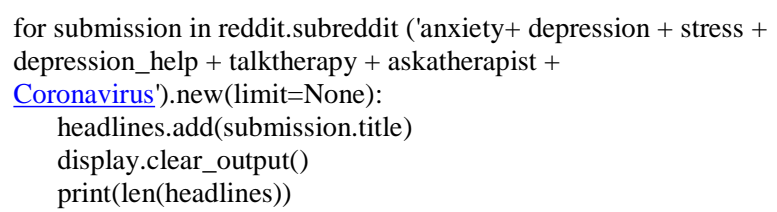

Figure 6. Collecting SubReddits from the Reddit website

The database is completely new, it is prepared especially for this chatbot because it includes questions and answers on issues of mental health such as depression and anxiety, whether in COVID-19 or not, as well as therapist's answers that can be beneficial. Figure 7 shows the first 5 negative and positive questions,

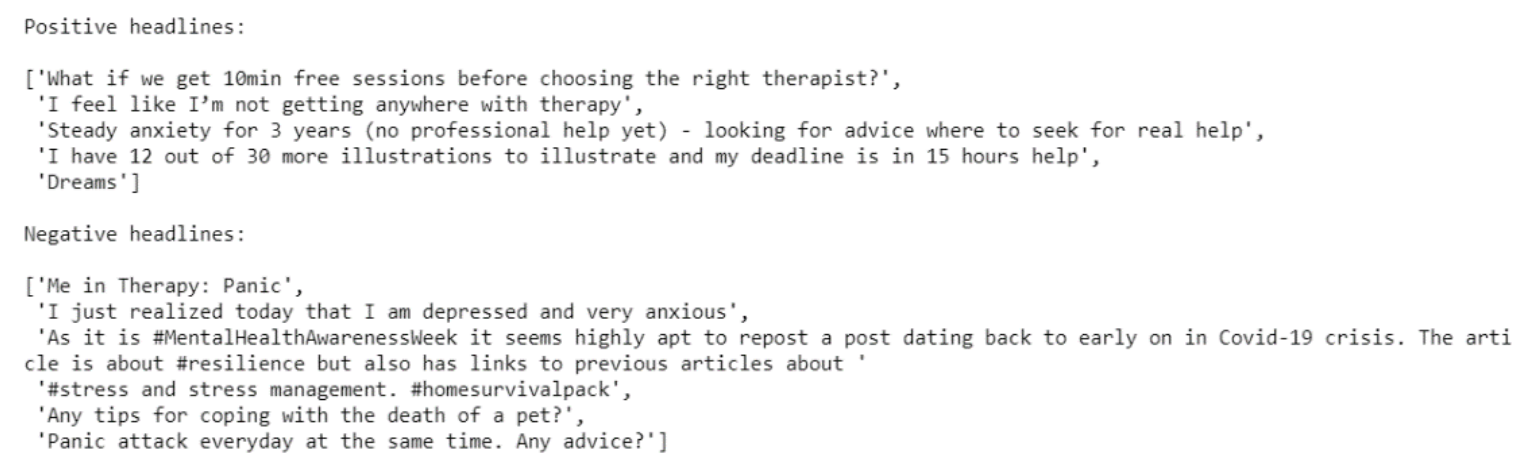

Figure 7. The first 5 positive and negative questions

After the execution of the code presented in Figure 6, 5735 questions were collected, of which $40.54 \%$ were negatives, $38.79 \%$ were neutral and $20.66 \%$ were positives. This is presented by the diagram in Figure 8. In this section, the verification of the frequencies of occurrence is necessary to validate the collected database. To validate it, it is necessary to have positive words. Because the chatbot has a depressed or anxious person in front of it, it must reassure him and lead a serious conversation with him. the pipeline steps of the natural language processing were followed and the calculation of words appearance frequencies is done. Table 1 shows the frequency of appearance of the most common negative and positive words in the collected questions. 


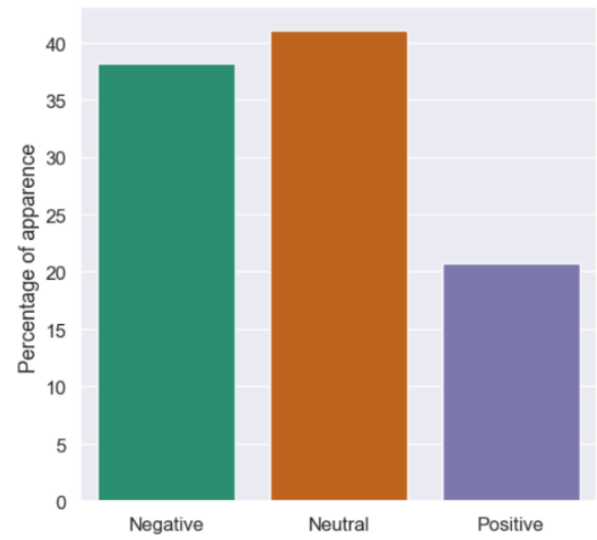

Figure 8. Question distribution of questions collected from Reddit

Table 1. Word appearance frequencies

\begin{tabular}{lclc}
\hline Word & $\begin{array}{c}\text { Positive Titles } \\
\text { Appearance Frequency }\end{array}$ & \multicolumn{1}{c}{ Word } & $\begin{array}{c}\text { Negative Titles } \\
\text { Appearance Frequency }\end{array}$ \\
\hline Help & 242 & Stress & 232 \\
Like & 160 & Depression & 198 \\
Therapist & 123 & Anxiety & 184 \\
Feel & 114 & Therapist & 166 \\
Need & 82 & Feel & 153 \\
Therapy & 76 & Life & 98 \\
Please & 68 & Get & 95 \\
Anxiety & 64 & Therapy & 86 \\
Get & 60 & Like & 86 \\
Anyone & 51 & Stressed & 85 \\
Know & 50 & Want & 77 \\
Someone & 47 & Bad & 76 \\
Friend & 46 & Anyone & 72 \\
Life & 45 & Feeling & 69 \\
Love & 44 & Know & 66 \\
Good & 42 & Depressed & 65 \\
Best & 41 & Stop & 63 \\
Better & 40 & Help & 57 \\
Stress & 39 & Else & 56 \\
One & 36 & People & 55 \\
\hline
\end{tabular}

Figures 9 and 10 show the frequency distributions of the most commonly used words in the positive and negative sets, respectively. The frequency of appearance of positive words is more important than negative words. This is extremely valuable, considering that the chatbot must carry on a positive conversation and thus has to express itself in a way that makes it feel comfortable for the person facing it. After completing the steps of the natural language processing unit, we moved on to the natural language comprehension unit and trained our data through the sequence-to-sequence re-information model built using the TensorFlow framework [43] and the Keras API [44]; with 50 epochs. Figures 11, 12 and 13 show the results of the training of our model are for epochs 1,5 , and 50.

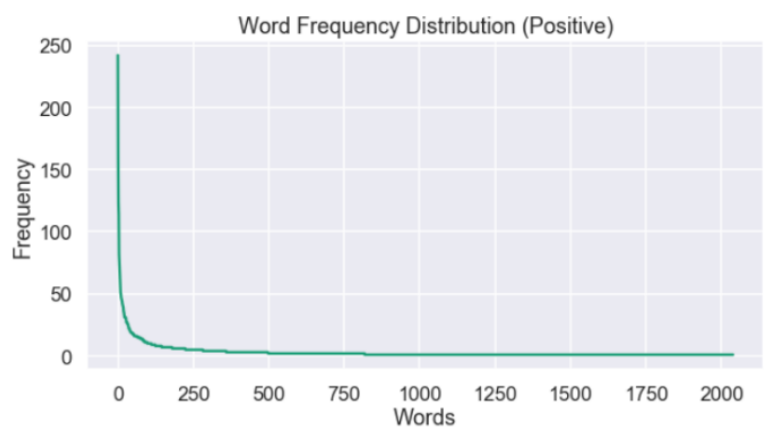

Figure 9. Frequency distribution of the appearance of positive words

Towards developing a pocket therapist: an intelligent adaptive psychological support ... (Intissar Salhi) 


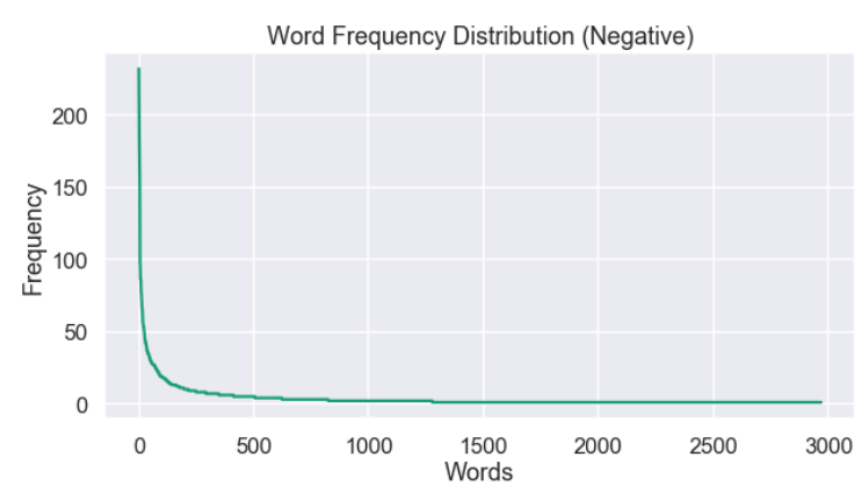

Figure 10. Frequency distribution of the appearance of negative words

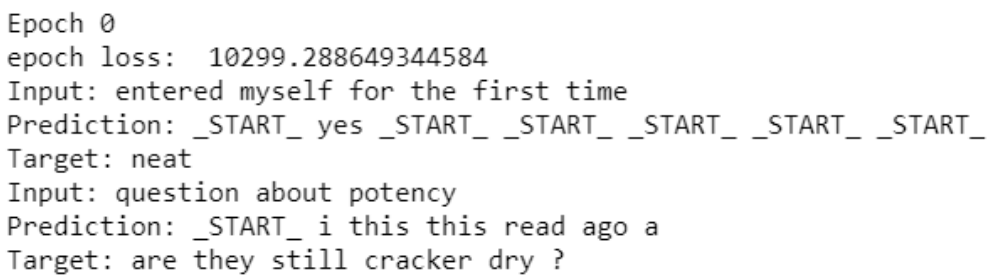

Figure 11. Training in epoch 1

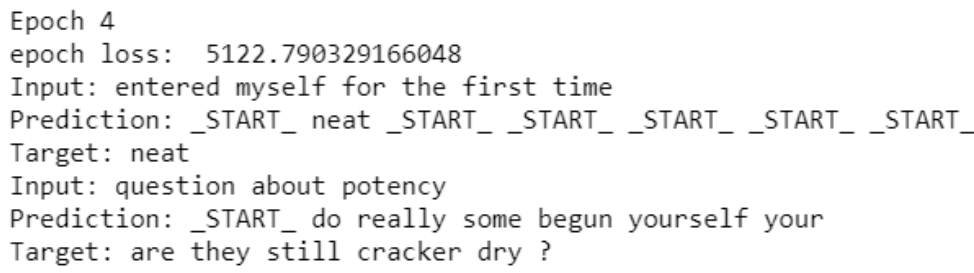

Figure 12. Training in epoch 5

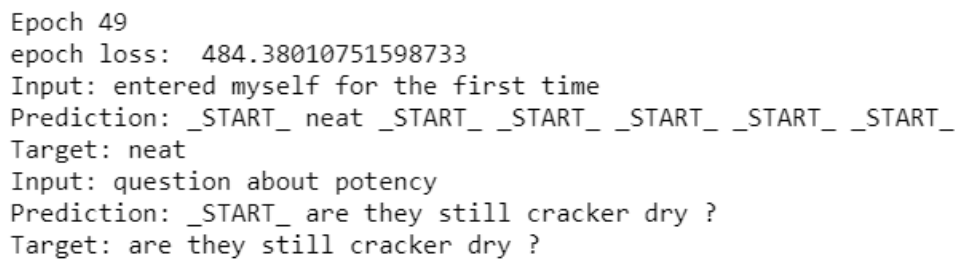

Figure 13. Training in epoch 50

The model has an accuracy of $95 \%$, this is calculated using the (5),

$$
\text { Accuracy }=1-\text { Loss }
$$

The comparison between the predictions and the targets is more interesting, if both are the same it means that the chatbot is performing properly. This justifies the decision to display them on the screens. chatbots that treat specific mental health in the context of a pandemic such as the one related to COVID-19. The others are more general. The approach treats emotions, while the others do not. Despite all this, we tested some systems with the collected database, the results were different but not very far from ours. this can be explained by the fact that these systems are not designed in the same context and do not take emotions into account. After 
training our model, we integrated the natural language comprehension unit into a therapist chatbot that we have implemented. Figure 14 shows the graphical interface of the therapist chatbot. We were able to build a chatbot that answers our initial problem, it can conduct a dialogue with a person in a fluid and clear way. The chatbot's answer is in the form of text and voice. This can facilitate interaction with the chatbot. The main concern that occurs is that he does not respond in the same way to messages he has never seen before.
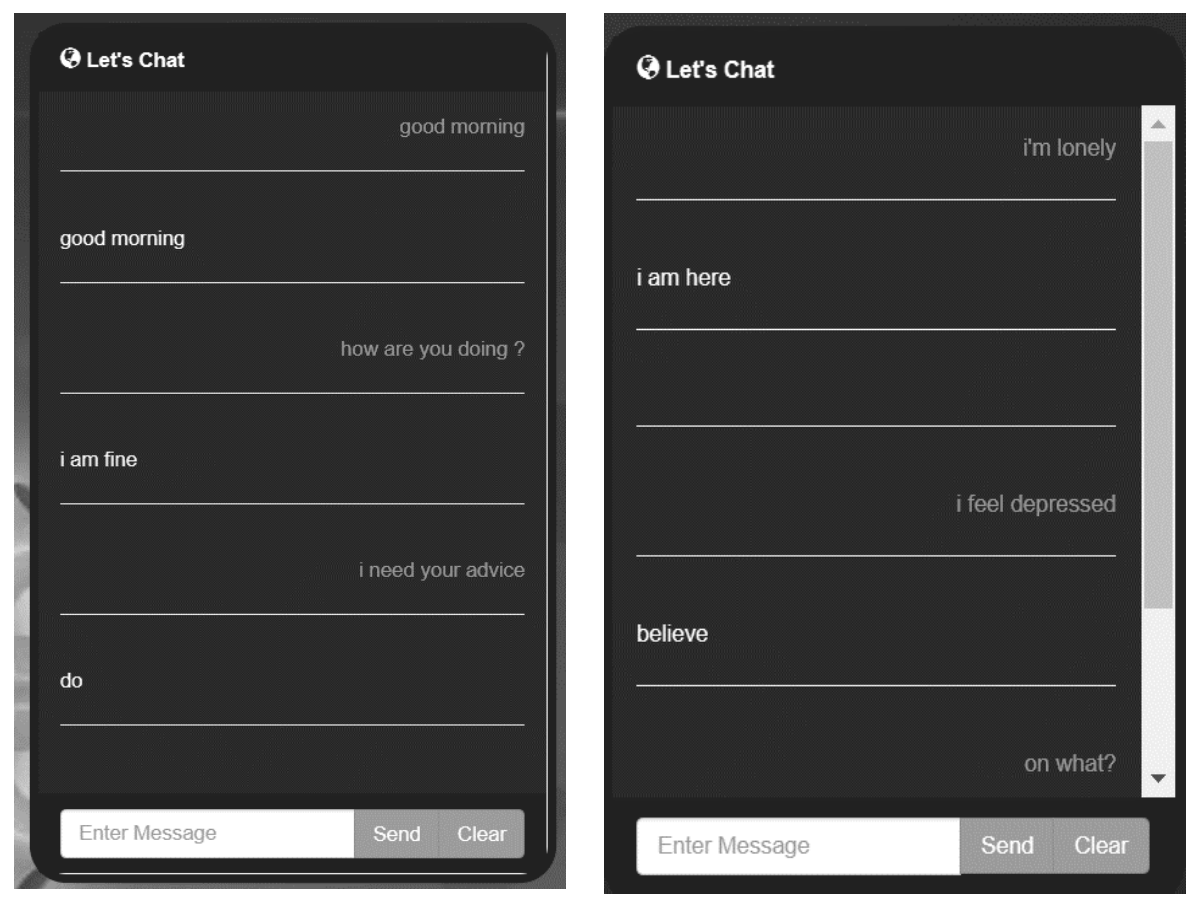

Figure 14. The graphical interface of the therapist chatbot

\section{CONCLUSION}

In this paper, we have tried to develop a chatbot system based on the sequential attention mechanism based on deep recurrent neural networks for psychological queries, to help people affected by mental health disorders, especially, students in COVID-19 pandemic situation. We proposed an advanced architecture of an adaptive therapeutic chatbot with self-learning abilities. This was designed with the principal objective to fill in a healthcare gap in the COVID-19 pandemic situation and to provide a flexible chat interface to answer questions from people who feel overwhelmed by the events. The Seq2Seq mode presented in the paper was built using the TensorFlow framework and the Keras API. The performance of the model was limited due to the limited training; however, the paper shows the use of a databased approach in the construction of the chatbot. Significant results can be achieved both by using high-performance computational tools and by modifying the training hyperparameters. These will be taken into account in future studies. this includes also the optimization of the chatbot utilizing the most recent optimization methods which are the Garra Rufa optimization, the CWO, and the EHO optimization.

\section{ACKNOWLEDGEMENT}

This work is supported by the University Hassan II of Casablanca- Morocco and The National Scientific Research and Technology Center (CNRST) under the program "support program for scientific and technological research related to COVID-19", project "Elaboration of a psychological and pedagogical support platform".

\section{REFERENCES}

[1] R. Rodríguez-Rey, H. Garrido-Hernansaiz, and S. Collado, "Psychological Impact and Associated Factors During the Initial Stage of the Coronavirus (COVID-19) Pandemic Among the General Population in Spain," Frontiers in Psychology, vol. 11, p. 1540, 2020, doi: 10.3389/fpsyg.2020.01540. 
[2] L. Shanahan, et al., "Emotional Distress in Young Adults during the COVID-19 Pandemic: Evidence of Risk and Resilience from a Longitudinal Cohort Study," Psychological Medicine, pp. 1-10, 2020, doi: 10.1017/S003329172000241X.

[3] K. Shah, D. Kamrai, H. Mekala, B. Mann, K. Desai, and R. S. Patel, "Focus on Mental Health During the Coronavirus (COVID-19) Pandemic: Applying Learnings from the Past Outbreaks," Cureus, vol. 12, no. 3, 2020, doi: $10.7759 /$ cureus.7405.

[4] W. Cullen, G. Gulati, and B. D. Kelly, "Mental health in the COVID-19 pandemic," QJM: An International Journal of Medicine, vol. 113, no. 5, pp. 311-312, 2020, doi: 10.1093/qjmed/hcaa110.

[5] C.-Q. Zhang, E. Leeming, P. Smith, P.-K. Chung, M. S. Hagger, and S. C. Hayes, "Acceptance and Commitment Therapy for Health Behavior Change: A Contextually-Driven Approach," Frontiers in Psychology, vol. 8, 2018, doi: 10.3389/fpsyg.2017.02350.

[6] M.O. Ogundele, "Behavioural and emotional disorders in childhood: A brief overview for paediatricians," World Journal of Clinical Pediatrics, vol. 7, no. 1, pp. 9-26, 2018, doi: 10.5409/wjcp.v7.i1.9.

[7] R. C. Kessler and B. B. Üstün, "The World Mental Health (WMH) Survey Initiative version of the World Health Organization (WHO) Composite International Diagnostic Interview (CIDI)," International Journal of Methods in Psychiatric Research, vol. 13, no. 2, pp. 93-117, 2004, doi: 10.1002/mpr.168.

[8] D. V. Vigo, et al., "A partnership for transforming mental health globally," The Lancet Psychiatry, vol. 6, no. 4, pp. 350-356, 2019, doi: 10.1016/S2215-0366(18)30434-6.

[9] C. O. Akosile, U. G. Mgbeojedo, F. A. Maruf, E. C. Okoye, I. C. Umeonwuka, and A. Ogunniyi, "Depression, functional disability and quality of life among Nigerian older adults: Prevalences and relationships," Archives of Gerontology and Geriatrics, vol. 74, 39-43, 2018, doi: 10.1016/j.archger.2017.08.011.

[10] P. Astuti, K. Kusnanto, and F. D. Novitasari, "Depression and functional disability in stroke patients," Journal of Public Health Research, vol. 9, no. 2, pp. 169-171, 2020, doi: 10.4081/jphr.2020.1835.

[11] J. R. Asarnow and D. Ougrin, "Editorial: Suicide and self-harm: advancing from science to preventing deaths," Journal of Child Psychology and Psychiatry, vol. 60, no. 10, pp. 1043-1045, 2019, doi: 10.1111/jcpp.13131.

[12] C. Oppenheimer, J. Silk, N. Jones, and R. Butterfield, "Neural Processing of Social Rejection and Suicide Risk," Biological Psychiatry, vol. 87, no. 9, S420, 2020, doi: 10.1016/j.biopsych.2020.02.1072.

[13] N. Weye, et al., "Association of Specific Mental Disorders With Premature Mortality in the Danish Population Using Alternative Measurement Methods," JAMA Network Open, vol. 3, no. 6, e206646, 2020, doi: 10.1001/jamanetworkopen.2020.6646.

[14] Tankovska, I. Memedi, and D. Dimitrovski, "Coronavirus Covid-19 Disease, Mental Health and Psychosocial Support," Society Register, vol. 4, no. 2, pp. 33-48, 2020, doi: 10.14746/sr.2020.4.2.03.

[15] N. A. Megahed and E. M. Ghoneim, "Antivirus-built environment: Lessons learned from Covid-19 pandemic," Sustainable Cities and Society, vol. 61, 102350, 2020, doi: 10.1016/j.scs.2020.102350.

[16] A. N. Vaidyam, H. Wisniewski, J. D. Halamka, M. S. Kashavan, and J. B. Torous, "Chatbots and Conversational Agents in Mental Health: A Review of the Psychiatric Landscape," Canadian Journal of Psychiatry, vol. 64, no. 7, pp. 456-464, 2019, doi: 10.1177/0706743719828977.

[17] A. A. Abd-alrazaq, M. Alajlani, A. A. Alalwan, B. M. Bewick, P. Gardner, and M. Househ," An overview of the features of chatbots in mental health: A scoping review," International Journal of Medical Informatics, vol. 132, 103978, 2019, doi: 10.1016/j.ijmedinf.2019.103978.

[18] A. A. Abd-Alrazaq, A. Rababeh, M. Alajlani, B. M. Bewick, and M. Househ,” Effectiveness and safety of using chatbots to improve mental health: Systematic review and meta-analysis," Journal of Medical Internet Research, vol. 22, no. 7, e16021, 2020, doi: 10.2196/16021.

[19] M. McTear, "Conversational AI: Dialogue Systems, Conversational Agents, and Chatbots," Synthesis Lectures on Human Language Technologies, vol. 13, no. 3, pp. 1-251, 2020, doi: 10.2200/s01060ed1v01y202010hlt048.

[20] E. H. Almansor and F. K. Hussain, "Survey on Intelligent Chatbots: State-of-the-Art and Future Research Directions," in Advances in Intelligent Systems and Computing, Springer Verlag, pp. 534-543, 2020, doi: 10.1007/978-3-030-22354-0_47.

[21] A. B. Kocaballi, et al., "The personalization of conversational agents in health care: Systematic review," Journal of Medical Internet Research, vol. 21, no. 11, e15360, 2019, doi: 10.2196/15360.

[22] S. Hobert and R. M. von Wolff, "Say Hello to Your New Automated Tutor - A Structured Literature Review on Pedagogical Conversational Agents," Wirtschaftsinformatik 2019 Proceedings, 2019.

[23] R. Bavaresco, et al., "Conversational agents in business: A systematic literature review and future research directions," Computer Science Review, vol. 36, 100239, 2020, doi: 10.1016/j.cosrev.2020.100239.

[24] A. O. Ikumoro and M. S. Jawad, "Intention to Use Intelligent Conversational Agents in e-Commerce among Malaysian SMEs: An Integrated Conceptual Framework Based on Tri-theories including Unified Theory of Acceptance, Use of Technology (UTAUT), and T-O-E," International Journal of Academic Research in Business and Social Sciences, vol. 9, no. 11, pp. 205-235, 2019, doi: 10.6007/ijarbss/v9-i11/6544.

[25] A. Heikkilä, "Natural Language Processing Techniques in Chatbot Development: How does a Chat-Bot Process Language?," Master Thesis, University of Jyväskylä, Jyväskylän yliopisto, Finlandia, 2020".

[26] M. Shumanov and L. Johnson, "Making conversations with chatbots more personalized," Computers in Human Behavior, 106627, 2020, doi: 10.1016/j.chb.2020.106627.

[27] I. V. Serban, et al., “A Deep Reinforcement Learning Chatbot,” arXiv preprint arXiv:1709.02349, 2017. 
[28] K. Palasundram, K. Palasundram, N. M. Sharef, N. Nasharuddin, K. Kasmiran, and A. Azman, "Sequence to Sequence Model Performance for Education Chatbot," International Journal of Emerging Technologies in Learning (IJET), vol. 14, no. 24, pp. 56-68, 2019, doi: 10.3991/ijet.v14i24.12187.

[29] E. H. Almansor and F. K. Hussain, "Survey on Intelligent Chatbots: State-of-the-Art and Future Research Directions," in Advances in Intelligent Systems and Computing, Springer Verlag, pp. 534-543, 2020, doi: 10.1007/978-3-030-22354-0_47.

[30] J. Weizenbaum, "ELIZA-A computer program for the study of natural language communication between man and machine," Communications of the ACM, vol. 9, no. 1, pp. 36-45, 1966, doi: 10.1145/365153.365168.

[31] R. Epstein, G. Roberts, and G. Beber, "Parsing the Turing Test: Philosophical and methodological issues in the quest for the thinking computer," Springer, Netherlands, 2009, doi: 10.1007/978-1-4020-6710-5.

[32] O. V. Deryugina, "Chatterbots," Scientific and Technical Information Processing, vol. 37, no. 2, pp. 143-147, 2010, doi: 10.3103/S0147688210020097.

[33] B. Inkster, S. Sarda, and V. Subramanian, "An empathy-driven, conversational artificial intelligence agent (Wysa) for digital mental well-being: Real-world data evaluation mixed-methods study," JMIR MHealth and UHealth, vol. 6, no. 11, e12106, 2018, doi: 10.2196/12106.

[34] Z. Safi, A. Abd-Alrazaq, M. Khalifa, and M. Househ, "Technical Aspects of Developing Chatbots for Medical Applications: Scoping Review," Journal of Medical Internet Research, vol. 22, no. 12, e19127, 2020, doi: 10.2196/19127.

[35] N. Ouerhani, A. Maalel, H. Ben Ghézala, and S. Chouri, "Smart Ubiquitous Chatbot for COVID-19 Assistance with Deep learning Sentiment Analysis Model during and after quarantine," Research Square, 2020, doi: 10.21203/rs.3.rs-33343/v1.

[36] A. Fadhil and G. Schiavo, "Designing for Health Chatbots," arXiv preprint arXiv:1902.09022, 2019.

[37] Y. Guo, Z. Hao, S. Zhao, J. Gong, and F. Yang, "Artificial intelligence in health care: Bibliometric analysis," Journal of Medical Internet Research, vol. 22,no. 7, e18228, 2020, doi: 10.2196/18228.

[38] B. Saberi and S. Saad, "Sentiment analysis or opinion mining: A review," International Journal on Advanced Science, Engineering and Information Technology, vol. 7, no. 5, pp. 1660-1666, 2017, doi: 10.18517/ijaseit.7.5.2137.

[39] J. D. Lafferty, A. McCallum, and F. C. N. Pereira, "Conditional Random Fields: Probabilistic Models for Segmenting and Labeling Sequence Data," ICML '01: Proceedings of the Eighteenth International Conference on Machine Learning, 2001, pp. 282-289.

[40] D. A. Permatasari, H. Fakhrurroja, and C. Machbub, "Human-Robot Interaction Based on Dialog Management Using Sentence Similarity Comparison Method," International Journal on Advanced Science, Engineering and Information Technology, vol. 10, no. 5, pp. 1881-1888, 2020, doi:10.18517/ijaseit.10.5.7606.

[41] O. I. Abiodun, A. Jantan, A. E. Omolara, K. V. Dada, N. A. E. Mohamed, and H. Arshad, "State-of-the-art in artificial neural network applications: A survey," Heliyon, vol. 4, no. 11, 2018, doi: 10.1016/j.heliyon.2018.e00938.

[42] I. Sutskever, O. Vinyals, and Q. V. Le, "Sequence to Sequence Learning with Neural Networks," Advances in Neural Information Processing Systems, pp. 3104-3112, 2014.

[43] TensorFlow. [Online]. Available: https://www.tensorflow.org/?hl=fr. Accessed: Feb. 232021.

[44] K. Team, Simple. Flexible. Powerful, Keras. [Online]. Available: https://keras.io/. Accessed: Feb. 232021. 